\title{
Defensive burying by mice: Intraspecific genetic variation and retention
}

\author{
DAVID B. HARDER and JOHN C. MAGGIO \\ Florida State University, Tallahassee, Florida
}

\begin{abstract}
Genotypically based within-species differences in defensive burying were examined in 180 mice representing 15 inbred strains. Each mouse was tested twice in a cylindrical test chamber containing two similar prods. In the first test, one of the prods was electrified, whereas in the second test (24 h later), neither prod was. Although most strains selectively buried the shock prod in the first test (as determined by bedding-height-at-prod and position-of-highest-beddingpile criteria), some strains did not discriminate between the shock and dummy prods and still others displayed little prod-directed bedding displacement at all (thereby resembling a heterogeneous nonshocked control group). In general, burying tended to be somewhat reduced in the second test, but strain differences in retention were observed. Factors contributory to the observed differences among strains and the need for multiple measures of burying are discussed. Collectively, these findings indicate that intraspecific genetic variation, acting at multiple burying-relevant behavioral levels, can be an important determinant of the expression of the defensive-burying response in mice.
\end{abstract}

The novel rodent behavior termed "defensive burying" by Pinel and Treit (1978) has been the focus of a considerable amount of research effort in the last few years. This behavior, first noted by Hudson (1950), is characterized by the persistent approach by some rodents to a localized, stationary source of aversive stimulation, and the covering of the source with materials found in the vicinity. A single exposure to the aversive stimulus is generally sufficient to initiate burying (Pinel, Treit, $\&$ Wilkie, 1980; Tarte \& Oberdieck, 1982), and the behavior often continues despite additional exposures encountered in the process of burying (this lab). Two general types of procedure have been employed to study this behavior. One uses a single aversive stimulus, and comparisons are made with independent control groups. The other uses two stimuli differing to some extent in aversive, visual, spatial, or other properties. In this second situation, within-subject comparisons of behavior toward each stimulus are possible.

Much of the work to date has examined the effects of manipulating various environmental conditions (Arnaut \& Shettleworth, 1981, Davis, Whiteside, Dickson, Thomas, \& Heck, 1981, and Pinel, Treit, Ladak, \& MacLennan, 1980, are some representative examples). Relatively few studies, however, have involved comparisons between species and/or between strains within a species (Davis, Whiteside, Heck,

The authors wish to thank Glayde Whitney, for support throughout this study, and Debbie Brock, for expert assistance in the preparation of the manuscript. Reprint requests should be sent to David B. Harder, Department of Psychology, Florida State University, Tallahassee, Florida 32306.
Dickson, \& Tramill, 1981; Maggio \& Harder, 1983; McKim \& Lett, 1979; Tarte \& Oberdieck, 1982; Treit, Terlecki, \& Pinel, 1980; Whillans \& Shettleworth, 1981). Furthermore, all but two (Maggio \& Harder, 1983; Treit et al., 1980) compared only two genetically distinct groups. Although such small numbers of groups provide a very limited basis for assessing the influence of genetic differences on defensive burying, within- and/or between-species burying differences were demonstrated in each.

Within-species strain comparisons of defensive burying have been reported only for rats (Rattus norvegicus) and mice (Mus musculus). McKim and Lett (1979) found that Long-Evans hooded rats displayed more preshock burying of a prod than did Sprague-Dawley albino rats. Tarte and Oberdieck (1982) found that Long-Evans hooded rats also spent more time burying a single shock rod than did Wistar albino rats, but only after habituation to the test chamber. Treit et al. (1980) reported, however, that in a two-prod situation Wistar rats spent more time burying than did Long-Evans rats and that Fischer rats buried more than either. Treit et al. (1980) also reported that CF-1-strain mice spent more time burying than did either CD-1 or BALB mice and, in contrast to the rat findings, that one strain (BALB) did not discriminate between the shock and control prods. Maggio and Harder (i983) reported that burying differences observed among four sublines of two strains of mice (DBA/1Bg and C57BL/10Bg) interacted strongly with length of the test chamber. Furthermore, a genotype-dependent abolition of burying was found when the chamber length was increased. These experiments suggest that within-species genetic variation may be an important factor affecting 
defensive burying performance. The two studies testing mice are particularly indicative because in each, an almost complete absence of burying was found for some genotypes.

A more complete picture of the generality of burying behavior within a species, and its potential variability due to genetic differences, might be obtained from comparisons of a considerably larger number of strains. The many commercially available inbred strains of mice provide a pool of subjects from which a suitably large number of comparison groups can be easily drawn. Since each strain has been inbred for at least 20 generations (some more than 100 generations), mice of the same sex within each strain are considered to be genetically identical. Withinstrain variation in burying performance can thus be attributed to environmental sources, whereas burying differences among strains reflect genetic differences (including genotype $\times$ environment interactions).

In conjunction with the investigation of intraspecific genetic variation, the present study also addresses some recently proposed methodological concerns. Modaresi (1982) has argued that certain aspects of the test chambers commonly employed in defensiveburying experiments may bias the results obtained. Modaresi showed that during four preshock habituation periods, hooded rats selectively pushed bedding toward the front half of a chamber (in which the front wall served as the door), regardless of the location of a prod. This tendency was also noticeable in a postshock test, but was found to interact with prod location during habituation. These findings demonstrate an effect on burying of a predisposition for oriented bedding displacement that is based on environmental features other than the intended aversive stimulus. Unfortunately, most of the test chambers previously used incorporate such potentially biasing features to some extent. Test chambers are usually rectangular (and therefore have corners); sometimes one or more sides are of a different material from the rest, and sometimes one side serves as a door. Since one or more sides are also frequently transparent, the potential for external visual stimulus differences affecting burying also exists. Such features predicate that the walls of the chamber are not uniform with respect to an aversive object such as a shock prod. Any tendency to treat one portion of the chamber differently from another based on such nonuniformities could influence defensive burying behavior.

One means by which these potential problems might be avoided is the use of an opaque, cylindrical, test chamber opening from the top. In such a test chamber, the prod (or other aversive stimulus) would constitute the sole variation on an otherwise uniform wall. Davis, Moore, Cowen, Thurston, and Maggio (1982) recently used a cylindrical chamber to demonstrate defensive burying by gerbils (Meriones un- guiculatus), but they and other investigators (Davis, Whiteside, Heck et al., 1981; Treit et al., 1980) were unable to demonstrate burying by gerbils when standard rectangular chambers were used.

A second methodological concern is the adequacy of the usual dependent measures of defensive burying, duration of burying, and bedding height at prod. Modaresi (1982) gives as a primary objection to these measures the ambiguity that remains concerning (1) the direction of bedding displacement activity considered to be defensive burying, (2) the positions of bedding piles relative to the aversive object, and (3) the extent to which the aversive object is actually covered. Strictly defined height-at-prod measures (which have been used in some studies) and positionof-bedding-pile measures, along with explicit a priori criteria for determining the occurrence of defensive burying, may reduce these ambiguities.

In addition to comparing a large number of inbred mouse strains, the present study, while addressing the methodological issues mentioned above, also examines the retention of the defensive-burying response in mice. Long retention periods have been demonstrated in rats (Beninger, MacLennan, \& Pinel, 1980; Pinel \& Treit, 1978; Pinel, Treit, \& Wilkie, 1980; but cf. Davis, Whiteside, Dickson et al., 1981) but have not, as yet, been examined in other species.

\section{METHOD}

\section{Subjects}

A total of 180 adult mice (Mus musculus) from 15 inbred strains were tested (see Figure 3 for the specific strains used). Ten males of each strain plus 10 females of one strain (C57BL/ 6J) served as subjects in the shock-treatment condition (experimental group). An additional 20 males (1 or 2 each from 13 strains) comprised a nonshocked control group. All mice were purchased from Jackson Laboratory (Bar Harbor, Maine) and were 80-120 days old when tested. Upon arrival, the mice were individually housed in $10 \times 24 \times 13 \mathrm{~cm}$ wire-bottomed metal cages, in temperature/humidity controlled rooms, on a 12:12-h light:dark cycle. Food and water were available ad lib in the home cages.

Prior to the present experiment, the mice were used in two studies not involving defensive burying or electric shock. In the first study, each male was placed in a plastic cage, containing wood-chip bedding (Beta-chip hardwood laboratory bedding, Northeastern Products Corp., Warrensburg, N.Y.), with one of the C57BL/6J females, for a 3-min period, during which ultrasonic vocalizations were monitored. In the second study, the mice were given two-bottle preference tests of a phenylthiocarbamide (PTC) or sodium cyclamate solution versus distilled water, following lithium-chloride-induced taste-aversion conditioning to that solution. The conditioning and testing in this study were conducted in the wire-bottomed home cages described above. Exposure to the bedding in the first study provided the mice some familiarity with the burying material. This appears to be the only direct connection between these two experiments and the present study, but effects of other treatment aspects, such as the tasteaversion conditioning, cannot be entirely ruled out. As the mice were purchased for use in these two studies without regard to defensive burying propensity, the 15 strains may be considered, for purposes of the present experiment, to approximate a random sample of strains commercially available from Jackson Laboratory. 


\section{Apparatus}

The mice were tested in two identical cylindrical metal chambers (20 cm diam $\times 18 \mathrm{~cm}$ high) with removable wire-mesh lids. Each chamber contained two prods positioned diametrically opposite one another on the chamber wall. Each prod consisted of four parallel wires embedded in, and extending through, a small rubber stopper mounted in a hole centered $3.25 \mathrm{~cm}$ above the metal floor. The wires of each prod were held apart at the distal end by a small circular Teflon insulator. The stoppers were mounted flush with the wall so that the wires of the prods extended horizontally $3.5 \mathrm{~cm}$ into the chamber. Prods used to deliver shocks were connected externally to a shock generator (Grason-Stadler Model E6070B, Concord, Mass.) set to deliver $2.0 \mathrm{~mA}$ whenever two adjacent wires were simultaneously contacted.

\section{Procedure}

All testing was done during the light portion of the light:dark cycle in a dimly lit $\left(0.69 \mathrm{~cd} / \mathrm{m}^{2}\right)$ room separate from the rooms in which the mice were housed. Only one mouse occupied the test room at any given time. Prior to each test, fresh bedding was spread evenly over the floor of the chamber to a depth of $2.0 \mathrm{~cm}$. Each subject in the experimental group was placed individually in a test chamber with one electrified (shock) prod and one nonelectrified (dummy) prod. Upon that subject's initial shock-prod contact, a 15-min test period was begun. This test-session length was chosen to be consistent with the existing literature on mouse defensive burying (Maggio \& Harder, 1983; Misslin \& Ropartz, 1981; Treit et al., 1980) and because unpublished pilot data had indicated that, if burying occurred at all, it occurred within a few minutes after contact with the shock prod. At the end of this period, the mouse was removed carefully from the chamber so as not to disturb the bedding in the process. Approximately $24 \mathrm{~h}$ later, each mouse was retested. The second test differed from the first in that (1) neither prod was connected to the shock source and (2) the 15-min test period began immediately after the mouse was placed in the chamber.

The nonshocked control-group mice were also given two tests separated by $24 \mathrm{~h}$. The testing procedure for these animals was identical to that used in the retest of the experimental group.

After all tests, the height of the bedding at the midline of the base of each prod was measured to the nearest $0.1 \mathrm{~cm}$. The position of the highest pile of bedding in the entire chamber was also determined. For this determination, a circular wire-mesh grid divided into $224(1.27 \mathrm{~cm} /$ side) squares, or partial squares (along the perimeter), was lowered horizontally into the chamber. The position of the highest pile of bedding was then recorded as the center of that grid square in which its highest point was located. Additionally, the time from placement of the mouse in the chamber to the initial shock-prod contact (shock latency) and the total duration of shock received (shock amount) were recorded for each experimental-group subject's first test.

Burying, in a strict definitional sense (Modaresi, 1982), requires that an object be covered up. Therefore, an a priori posttest criterion for burying was established. A mouse, or group of mice, was not considered to have buried a prod unless the height of bedding at the prod was at least equal to the height of the top of the prod (i.e., $3.5 \mathrm{~cm}$ ). Coverage of a prod resulting from burying behavior specifically directed at the prod should, however, be distinguished from coverage as a by-product of random digging activity. Bedding piles elsewhere in the chamber as high as or higher than the mound covering the prod indicate that nonprod-related activity by the mouse may have been sufficient to have raised the prod mound. Accordingly, a second burying criterion was adopted to minimize this problem. The highest point of bedding in the chamber was required to be at or near the prod. Specifically, it had to be within a $5.0-\mathrm{cm}$ radius of the base of the covered prod. This radius encompassed the entire prod and was intended to allow for some variation in the exact location of the highest point, while still requiring that the maximal bedding displacement be directed toward the prod.

Differential coverage of the shock and dummy prods by mice in the experimental group would be an indication that the shock in particular, not prods per se, elicited burying behavior. Mice in the control group would not be expected to show this discrimination. Thus, the difference between the bedding heights at the two prods for each mouse (difference score) was used, along with the position of highest point, as an indicant of discrimination between the prods.

For the height-at-prod measure, overall experimental versus control group and shock prod versus dummy prod comparisons were done via a two-factor ANOVA (repeated on prod type) followed by Tukey's HSD multiple comparisons. The distance-ofhighest-point means for the experimental and control groups were compared via an independent groups $t$ test. For each of the three measures (height at shock prod, difference score, and distance of highest point from shock prod), a single-factor, independent groups ANOVA was performed on individual first-test strain means within the experimental group. These analyses were also followed by Tukey's HSD multiple comparisons to determine specific strain differences and similarities. A combined buryingmeasure value, giving equal weight to each of the three measures above, was then calculated for each strain (see Appendix). A cluster diagram based on these combined values (drawn according to a maximum differences algorithm outlined by DeGhett, 1978) was used to compare the strains on overall burying performance.

\section{RESULTS}

\section{First Test}

The basic effect of the shock on prod coverage is evident in Figure 1. The mean bedding height at the shock prod for the experimental group exceeded the height of the prod itself, whereas the mean at the dummy prod and the means at both prods for the control group were all below the 3.5-cm-height criterion. An analysis of variance indicated significant main effects for treatment condition $[F(1,178)=8.47$, $\mathrm{p}<.001]$ and type of prod $[F(1,178)=14.04, p<.001]$, as well as a strong interaction between these factors $[F(1,178)=31.94, p<.001]$. One prod (P1, Figure 1) was randomly designated to be the shockprod analog for the control group in this ANOVA. Tukey's HSD multiple comparisons confirmed that the experimental group's mean height at the shock prod was significantly greater than the mean height at the dummy prod $[Q(4,318)=15.49, \mathrm{p}<.01]$ and

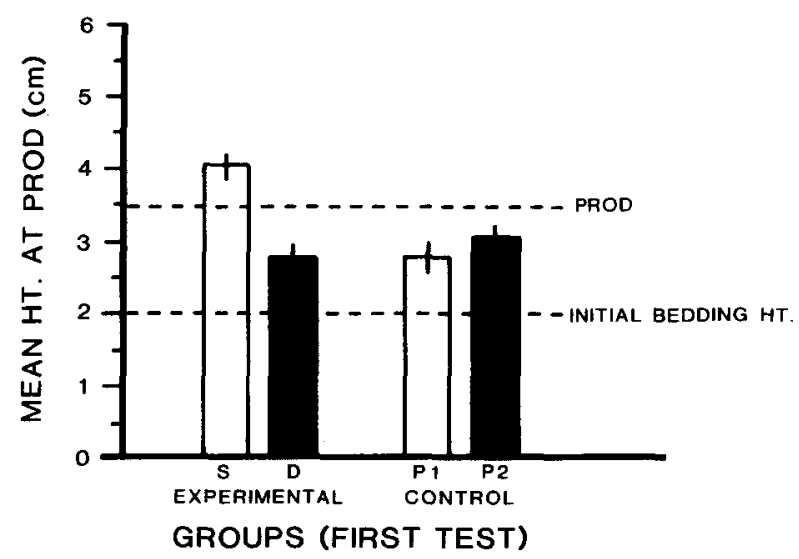

Figure 1. First-test mean bedding heights $( \pm$ SE) at the shock (S) and dummy (D) prods for the experimental group, and at Prod 1 (P1) and Prod 2 (P2) for the control group. 
was also greater than the means at either Prod 1 $[Q(3,178)=5.48, p<.01]$ or Prod $2[Q(2,178)=$ $4.37, \mathrm{p}<.01]$ for the control group. The mean heights at the two prods for the control group were not significantly different from each other $[Q(2,38)$ $=1.10, \mathrm{p}>.05]$ or from the mean height at the dummy prod for the experimental group $[Q(3,178)=$ $1.10, \mathrm{p}>.05]$.

The directionality of maximal bedding displacement by the experimental and control groups can be seen in Figure 2. The individual highest points tended to cluster near the shock prod in the experimental group but did not cluster near either prod in the control group. The mean distance of the highest points from the shock prod for the experimental group was within the $5.0-\mathrm{cm}$-criterion radius (mean $=4.86 \pm$ $.81 \mathrm{~cm}$ ). The mean distances from both controlgroup prods were well outside this criterion (Prod 1 mean $=14.43 \pm 1.25 \mathrm{~cm}$; Prod 2 mean $=10.75 \pm$ $1.30 \mathrm{~cm})$. A t test showed the experimental group mean distance to be significantly less than even the closer mean distance (Prod 2) for the control group $[\mathrm{t}(178)=4.11, \mathrm{p}<.001]$.

Although the experimental group as a whole selectively buried the shock prod according to the two burying criteria, substantial differences among the various strains comprising this group were found. Figure 3 presents ordered means for each of the 16 strains (note that the female C57BL/6J mice are treated here as a separate strain) on the height-atprod (3a), difference-score (3b), and distance-ofhighest-point $(3 \mathrm{c})$ measures. In each panel, the vertical lines just to the left of the strain names connect
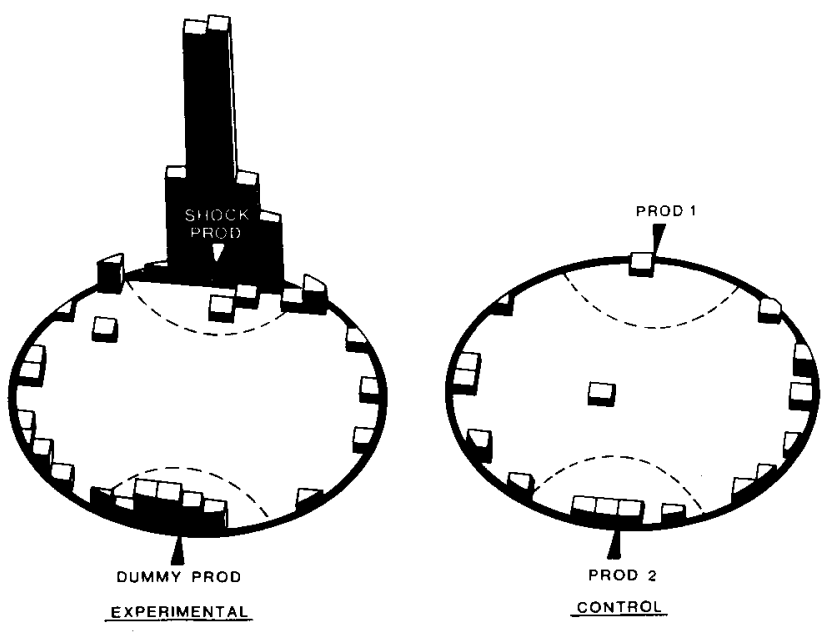

Figure 2. Positions of individual highest bedding points within the test chamber for the experimental and control groups on the first test. The number of mice whose highest point fell in a grid square is indicated by the height of the bar extending from that square (the largest bar represents 37 mice, the smallest represents 1 mouse). The dashed arcs indicate the $5.0-\mathrm{cm}$ criterion radii. strains found not to differ by Tukey's HSD multiple comparisons. Means for the control group are shown for comparison, but were not included in the strain analyses.

Figure $3 \mathrm{a}$ shows the mean bedding height at the shock and dummy prods for each strain. In all strains, the height at the shock prod (shaded + openbar sections) was greater than the height at the dummy prod (shaded section). However, considerable variation was observed among strains regarding both the absolute heights at the shock prod and the differences between the two prod means. A single-factor ANOVA performed on shock-prod heights revealed a significant main effect for strain $[F(15,144)=7.05$, $\mathrm{p}<.001]$. The particular strains differing on this measure (at $p<.01$ ) are indicated at the left of the panel by nonoverlap of the vertical lines.

Similarly, significant differences were found among strains for the difference-score (i.e., the height at shock prod minus the height at dummy prod) means shown in Figure $3 b[F(15,144)=3.24$, $\mathrm{p}<.001]$. Figure $3 \mathrm{c}$ presents strain means for the distance-of-highest-point-from-the-shock-prod measure. Here too, a significant overall strain effect was found $[F(15,144)=2.93, p<.005]$. Due to greater within strain variability, however, fewer individual strains differed significantly in the multiple comparisons on these latter two measures than on the height-at-shock-prod measure.

The actual location in the test chamber of the mean highest point for each strain is shown in Figure 4. For most strains, the mean position was found to be near the shock prod. In fact, 10 of 16 were within the 5.0$\mathrm{cm}$ criterion radius. In contrast, a few strains (e.g., A/J, 129/J, and NZB/BINJ) displayed poorer directionality of bedding displacement, with mean highest points located near the center of the chamber.

If the three measures used in Figure 3 (height, difference score, and distance) are valid estimators for particular aspects of defensive burying, one would expect a tendency for large height means to be associated with large difference-score means and with small distance means. These relationships were indeed found. Strain means on the height-at-shockprod measure were found to correlate positively with strain means on the difference-score measure $[r=.61$, Pearson's $R(14)=3.02, p<.01$ ]. The expected negative correlations of each of these two measures with the distance measure were also observed [height:distance $r=-.43, R(14)=1.84, p<.05$; difference score:distance $r=-.79, R(14)=4.92, p<.001]$. The substantial, but not perfect, correlations suggested that the three measures were related without being redundant and, therefore, that a combination of all three would provide a better basis for strain comparisons than any single measure alone. Therefore, a combined burying value giving equal weight to each of the three measures was calculated for each strain. 

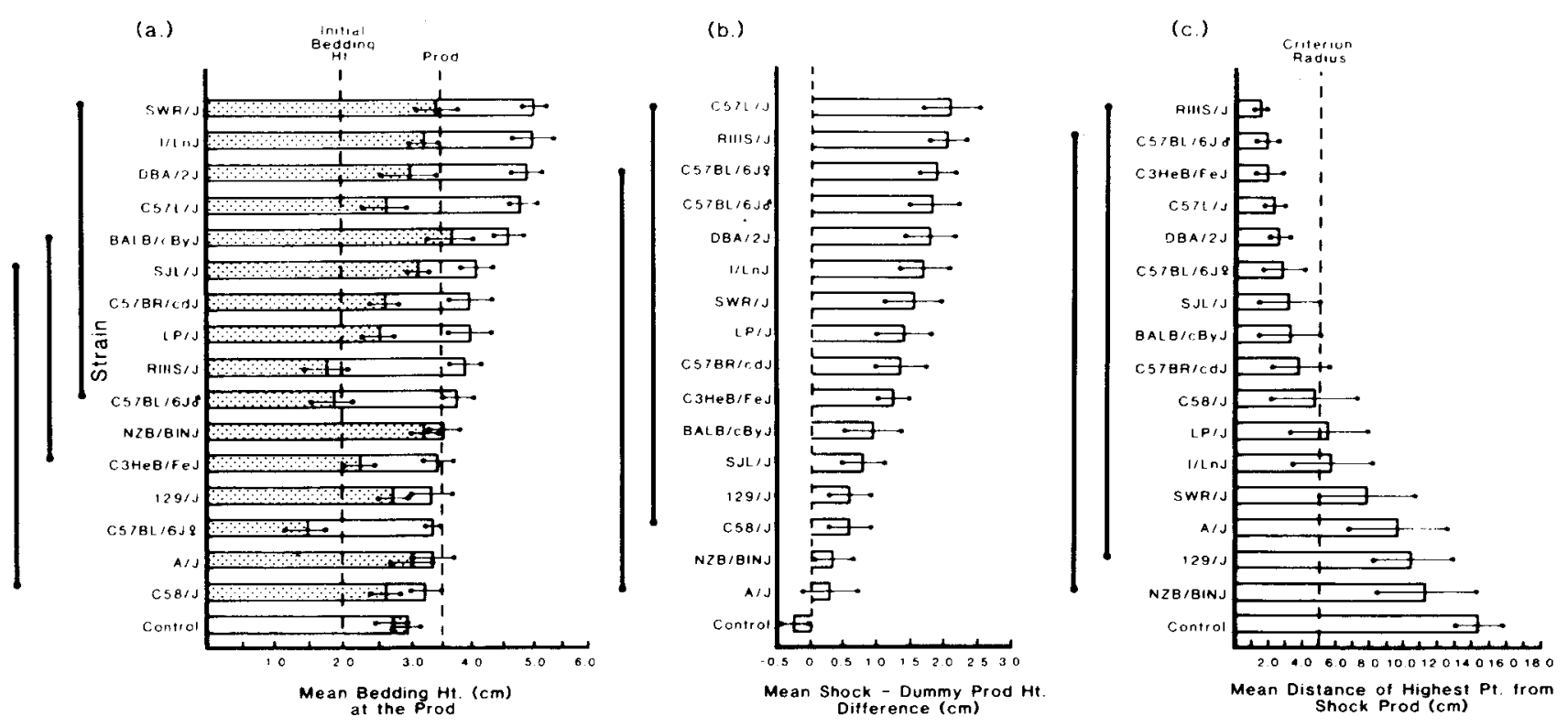

Figure 3. Ordered first-test strain means $( \pm S E)$ on the height-at-the-prod (a), difference-score (b), and distance-from-prod (c) measures. The vertical lines on the left of each panel connect strains not differing via multiple comparisons. In panel a, the shaded portions of the bars indicate mean heights at the dummy prod. The unshaded portions indicate mean heights at the shock prod.

For this combined value, each strain's height and difference-score means were converted to the distance-measure scale and then the average of the three means was taken (see Appendix for details).

Figure 5, a cluster diagram based on combined values, shows the relative differences in overall burying performance among the strains (and controls).

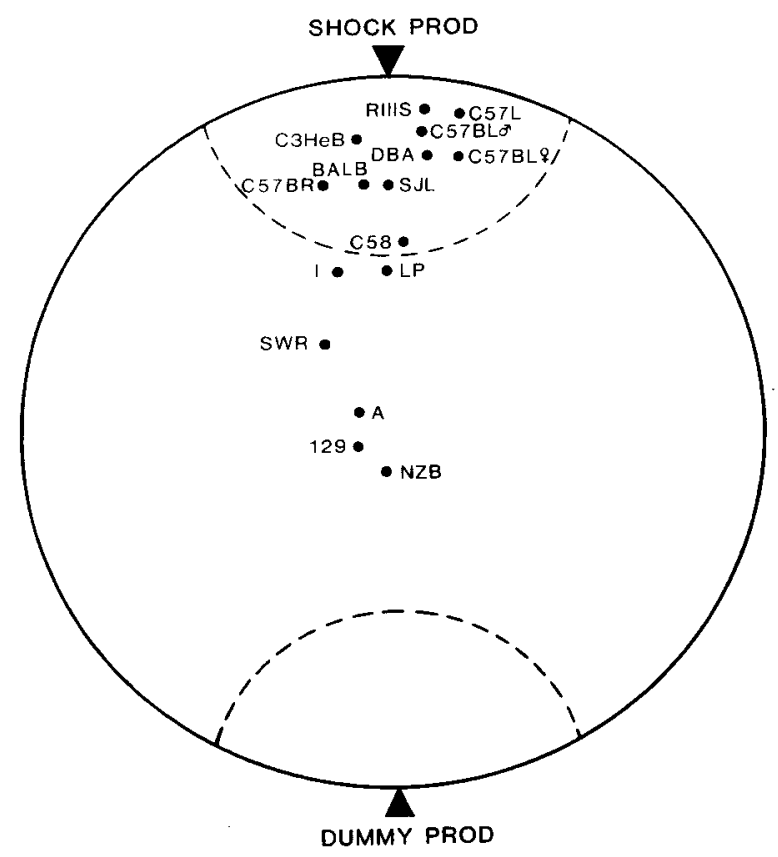

Figure 4. Strain mean highest point positions within the test chamber on the first test. The dashed arcs indicate the $5.0-\mathrm{cm}$ criterion radii.
Two primary strain clusters are evident, an upper cluster $(\mathrm{C} 57 \mathrm{~L} / \mathrm{J}$ to $\mathrm{SJL} / \mathrm{J})$ of relatively good buriers, and a lower cluster (C58/J to A/J, plus controls) of relatively poor buriers. The four strains in the lower cluster more closely resemble the nonshocked control group than they do the other shocked strains. Finer divisions based on smaller differences between clusters may also be made (e.g., C57L/J and DBA/2J vs. the rest of the upper cluster strains), but confidence in such distinctions necessarily decreases as the magnitude of the difference decreases.

Similar combined burying values among strains were sometimes achieved in dissimilar ways. The different patterns of behavior exhibited by the four strains in the lower cluster, all associated with relatively poor burying, are of particular interest. These patterns can most easily be seen in Table 1 . The upper cluster strains each had at least $80 \%$ of their highest bedding points in the prod areas (i.e., within the $5.0-\mathrm{cm}$ criterion radii), with a strong bias toward the shock prod area. Of the four lower cluster strains, two (A/J and NZB/BINJ) also had $80 \%$ or more in the prod areas, but here the highest points were divided almost equally between the shock and dummy prods. For these two strains, poor burying seems to have been a consequence of a lack of discrimination between the prods rather than a lack of prod-directed bedding displacement. (The fact that all but three mice of these strains covered one or the other of the prods supports this idea.) Bedding displacement by a third lower cluster strain $(129 / \mathrm{J})$, however, was not noticeably prod directed at all. Relatively few highest points fell within either prod area, a pattern of dispersion approaching the scat- 


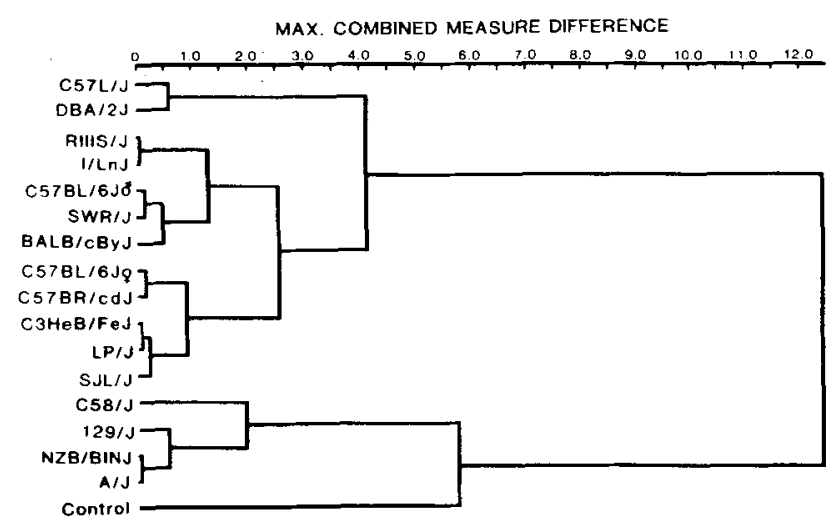

Figure 5. Clustering of strains on the combined buryingvalue measure. Combined values are ordered from best (top) to worst (bottom). The difference between the combined values of the two outermost strains of any cluster is indicated by the vertical farthest to the right that connects them (e.g., the difference between RIIIS/J and SJL/J is 2.55).

tered distribution found in the control group. In contrast to the three other lower cluster strains, the poor burying by the fourth strain $(\mathrm{C} 58 / \mathrm{J})$ resulted from a general lack of bedding displacement of any sort (although what little did occur was predominantly near the shock prod). Only three of these mice made a pile of bedding anywhere in the chamber of sufficient height (i.e., $\geqslant 3.5 \mathrm{~cm}$ ) to have covered a prod. Thus, at least three distinct factors can be identified as contributing to the similarly poor burying observing in these four strains: (1) nondiscrimination between shock and dummy prods, (2) lack of prod-directedness in bedding displacement, and (3) lack of bedding displacement in general.

Table 1

Percentage of Subjects with Highest Bedding Piles In and Outside of the Prod Areas for Individual Strains in the First Test

\begin{tabular}{|c|c|c|c|}
\hline \multirow[b]{2}{*}{ Strain } & \multicolumn{2}{|c|}{$\%$ In a Prod Area } & \multirow{2}{*}{$\begin{array}{c}\% \text { Outside } \\
\text { Either } \\
\text { Area }\end{array}$} \\
\hline & Shock & Dummy & \\
\hline C57L/J & 90 & 0 & 10 \\
\hline L:BA/2J & 90 & 0 & 10 \\
\hline RIIIS/J & 100 & 0 & 0 \\
\hline $\mathrm{I} / \mathrm{LnJ}$ & 70 & 20 & 10 \\
\hline C57BL/6J & 90 & 0 & 10 \\
\hline SWR/J & 60 & 30 & 10 \\
\hline BALB/cByJ & 90 & 10 & 0 \\
\hline C57BL/6Jo & 90 & 0 & 10 \\
\hline C57BR/cdJ & 80 & 0 & 20 \\
\hline $\mathrm{C} 3 \mathrm{HeB} / \mathrm{FeJ}$ & 90 & 0 & 10 \\
\hline $\mathbf{L P} / \mathbf{J}$ & 70 & 10 & 20 \\
\hline $\mathrm{SJL} / \mathrm{J}$ & 90 & 10 & 0 \\
\hline $\mathrm{C} 58 / \mathrm{J}$ & 80 & 20 & $\mathbf{0}$ \\
\hline $129 / \mathrm{J}$ & 30 & 20 & 50 \\
\hline NZB/BINJ & 40 & 40 & 20 \\
\hline $\mathbf{A} / \mathbf{J}$ & 50 & 40 & 10 \\
\hline Control & 5 & 20 & 75 \\
\hline
\end{tabular}

Since shocks were, in effect, self-administered by mice in the experimental group, there was a concern that burying differences among strains might reflect differences in the amount of shock received rather than genetic variation. The amount of shock received by the mice was generally small (overall group mean $=0.19 \mathrm{sec}$ ) with individual strain means ranging from 0.09 to 0.42 sec. No significant correlation of shock amount with means on the height, difference score, or distance measures was found, however $[R(14)=$ $-1.66, \mathbf{R}(14)=-0.05$, and $\mathbf{R}(14)=-0.91$, respectively, all ps $>.05]$. The total length of time spent in the test chamber was also controlled, to some extent, by the mice, since the 15 -min test period began only after the shock prod was first contacted. Despite considerable variation in shock latency among strains (overall group mean $=\mathbf{4 . 7 8} \mathrm{min}$, strain means range $=0.86$ to $15.29 \mathrm{~min}$ ), latency, too, was found not to be significantly correlated with any of the burying measures $[R(14)=-0.04, R(14)=0.36$, and $R(14)=$ -0.30 for height, difference score, and distance, respectively, all ps $>.05$ ].

\section{Retest}

Those portions of the circumference of the test chamber within $5.0 \mathrm{~cm}$ of a prod (i.e., within both prod areas) constituted about one-third of the entire circumference. Since nearly all highest points occurred at the chamber perimeter, about one-third would be expected to fall within the prod areas if the points were distributed randomly around the circumference. For the control group, $25 \%$ and $40 \%$ of the highest piles occurred within the prod areas on the first test and retest, respectively. For the experimental group, the percentage within the prod areas was much larger than expected by chance on both tests (first test $86 \%$, retest $70 \%$ ). The shock received in the first test thus continued to affect the behavior of the experimental group mice such that prod-oriented bedding displacement occurred in the retest. It was evident, however, that the experimental-group mice did not discriminate between the two prods in the retest (in which the shock was no longer available as a discriminative cue) as they had done in the first test. In the first test, $85 \%$ of the highest points within a prod area were in the shock-prod area and only $15 \%$ were in the dummy-prod area. In the retest, the highest points were evenly divided between the two areas $(53 \%$ and $47 \%)$. This lack of discrimination, while hardly surprising, since the prods were not distinct, made the measures used for analysis of the first-test results inappropriate for the retest because these measures assumed two distinct prod types (shock and dummy). Instead, the proportion of mice meeting both preestablished burying criteria at either prod was used for group and strain comparisons involving retest data.

Chi-square tests indicated that the proportion of 
Table 2

Percentage of Mice in Each Strain Meeting Both Burying Criteria (at Either Prod) in the First Test and Retest

\begin{tabular}{lcc}
\hline \multicolumn{1}{c}{ Strain } & First Test & Retest \\
\hline C57L/J & 80 & 50 \\
DBA/2J & 90 & 70 \\
RIIS/J & 80 & 10 \\
I/LnJ & 80 & 50 \\
C57BL/6J o & 70 & 10 \\
SWR/J & 80 & 70 \\
BALB/cByJ & 100 & 60 \\
C57BL/6J\& & 50 & 11 \\
C57BR/cdJ & 80 & 40 \\
C3HeB/FeJ & 50 & 20 \\
LP/J & 70 & 50 \\
SJL/J & 100 & 63 \\
C58/J & 20 & 40 \\
129/J & 20 & 20 \\
NZB/BINJ & 70 & 70 \\
A/J & 80 & 60 \\
Control & 20 & 15 \\
\hline
\end{tabular}

subjects meeting both criteria was significantly larger in the experimental group than in the control group on both the first test $\left(112 / 160\right.$ vs. $4 / 20, \chi^{2}=19.4$, $\mathrm{p}<.001)$ and the retest $\left(68 / 156\right.$ vs. $3 / 20, \chi^{2}=6.02$, $\mathrm{p}<.05)$. The proportion of experimental-group mice burying a prod in the retest, while exceeding that of the control group, was nevertheless substantially smaller than the proportion burying in the first test $\left(68 / 156\right.$ vs. $\left.112 / 160, \chi^{2}=51.82, p<.001\right)$. No difference between the control group proportions on the two tests was found $\left(3 / 20\right.$ vs. $4 / 20, \chi^{2}=0.31$, $\mathrm{p}>.05)$. These findings suggest some retention of the defensive burying response $24 \mathrm{~h}$ after experiencing the shock, but not complete retention.

The individual strain results presented in Table 2 revealed that retention of burying in the retest was not uniform across the strains of the experimental group. Some strains (e.g., RIIIS/J and C57BL/6J, males and females) virtually ceased burying in the retest. Others (e.g., DBA/2J and NZB/BINJ) showed little or no reduction in the proportion of mice meeting both criteria. Despite this variability, a significant correlation between percentage of subjects burying on the first test and on the retest was observed $[r=.56, R(14)=2.50, p<.025]$.

\section{DISCUSSION}

In attempting to address a variety of recently expressed methodological concerns surrounding defensive burying, several procedural modifications of the standard burying paradigm were adopted in the present experiment. First, by using a test chamber with a uniformly curved wall, and by introducing subjects into the chamber from the top, concerns regarding the potentially biasing influences of corners (Davis et al., 1982) and doors (Modaresi, 1982), re- spectively, on burying were diminished. Second, by specifying a priori that for a prod to be considered defensively buried (1) the height of burying material had to equal or surpass that of the prod, and (2) the location of the highest pile of bedding had to be near the prod, concerns regarding random displacement of burying material and lack of prod coverage (Modaresi, 1982) were taken into account. Third, by using multiple measures of burying, a more refined analysis of the various factors involved in the expression of this response was possible. Furthermore, by combining these individual measures into one overall value, we believe that a more valid assessment of the extent of burying can be made (i.e., the likelihood of measure-dependent errors in determining whether a subject or group did or did not bury is reduced).

These methodological questions aside, the large number of genotypically divergent groups examined in the present study provides a data base for assessing genetic contributions to defensive burying that is much broader than any previously reported. The results of this survey show (1) the frequency of the defensive burying response to aversive stimulation in many strains of mice, and (2) the potential variability of the response due to intraspecific genetic differences. (A few strains displayed essentially no defensive burying under the same test conditions in which nearly all individuals of other strains buried.) The present results also indicate that the observed differences in the display of the burying response may be attributable to a diverse set of genetically influenced factors. The observation that strains, when tested in a given environmental context, can independently vary on one or more of (1) shockprod/dummy-prod discrimination (difference-score measure), (2) burying magnitude (height-at-the-prod measure), (3) burying directionality (location-ofhighest-point measure), and (4) learning/retention (first-test vs. retest comparison) suggests that the display of the defensive-burying response may be subject to various effects of genotypic divergence (e.g., visual acuity, pain thresholds, motor patterns, memory, and integrative capacity, to name a few).

In addition to providing useful information regarding "strains of choice" for future mouse defensiveburying research in general, the results of the present experiment may also prove valuable in indicating those subjects especially suitable for delineating the genetic modes of transmission of the response. Comparison of the degree of genetic relatedness of particular strains with the similarity of their burying performance allows these subjects to be identified. Most promising perhaps is the great difference in burying between the C58/J strain and the three C57 strains (C57L/J, C57BL/6J, and C57BR/cdJ). These four strains belong to a large family of closely related strains and sublines about which information concerning specific genetic similarities and differences is 
comparatively abundant. It may, therefore, be possible to determine what genetic dissimilarities are associated with particular burying differences within this family.

The substantial defensive burying exhibited by some strains $24 \mathrm{~h}$ after receiving the shock provides evidence that a species other than rats can display the burying response as a result of one-trial learning and retention. Direct comparison of the present retentionbased burying levels for mice and those previously reported for rats is difficult, however, due to the numerous procedural differences involved. Furthermore, it has not been shown whether the retention levels reported in rat studies are specific to the one strain tested or are characteristic of rats in general. Our results demonstrate that even though two strains may show similar burying patterns on an initial test, they can be markedly different 24 h later.

The intraspecific and interspecific differences in defensive burying that have now been shown in several studies emphasize the hazards of drawing conclusions about the influence of environmental variables based on results from a single type of experimental subject. These same studies, however, also demonstrate that genetic variables do not operate in isolation either (e.g., the interaction of strain and retention interval shown here; the dependence of burying in gerbils on test-chamber shape demonstrated by Davis et al., 1982; the interaction of subline and chamber length shown in Maggio \& Harder, 1983; the shock-elicited burying difference between hooded and albino rats found by Tarte $\&$ Oberdieck, 1982, but not by McKim \& Lett, 1979; the interaction of strain and habituation period also shown in these latter two studies). Thus, the results of the present experiment must be considered in light of the environmental context in which they were obtained. In other testing situations (e.g., those employing rectangular test chambers, single-prod paradigms, or different aversive stimuli), it is entirely possible that the burying heirarchy herein demonstrated among inbred strains would be different. That BALB mice have previously been reported to display poor burying in a rectangular test chamber (Treit et al., 1980) but displayed relatively good burying under the present round test chamber conditions further supports this notion.

In conclusion, we suggest that the defensive-burying response to aversive stimulation should be viewed as an interactive product of genetic and environmental factors. As such, hypotheses regarding response generality (this report), its species-specificity as a defensive reaction (Pinel \& Treit, 1979), and its adaptive significance in nature (Whillans \& Shettleworth, 1981) must be tempered by the scarcity of available evidence relevant to these issues. Although genetic and environmental factors have been examined with domesticated rodent species in highly controlled situations, there have been very few reports pertinent to (and none directly addressing) whether defensive burying occurs in more naturalistic contexts. At best, field and laboratory observations (Owings, Borchert, \& Virginia, 1977; Owings \& Coss, 1978) suggest that behavior analogous to defensive burying may be used by ground squirrels (Spermophilus beecheyi) when confronted by snakes. Possibly related, the burial of dead conspecifics by rats, in response to cadaverine and putrescine, has also been demonstrated (Pinel, Gorzalka, \& Ladak, 1981). Thus, observations and experiments that directly assess the role (if any) of defensive burying in more naturalistic situations appear necessary before general conclusions regarding the above hypotheses can be drawn.

\section{REFERENCES}

Arnaut, L., \& Shetrteworth, S. J. The role of spatial and temporal contiguity in defensive burying in rats. Animal Learning \& Behavior, 1981, 9, 275-280.

Beninge R, R. J., Maclennan, A. J., \& Pinel, J. P. J. The use of conditioned defensive burying to test the effects of pimozide on associative learning. Pharmacology, Biochemistry \& Behavior, $1980,12,445-448$.

Davis, S. F., Moore, S. A., Cowen, C. L. , Thurston, D. K., \& Maggio, J. C. Defensive burying in the Mongolian gerbil (Meriones unguiculatus) as a function of size and shape of the test chamber. Animal Learning \& Behavior, 1982, 10, 516-520.

Davis, S. F., Whiteside, D. A., Dickson, V. A., Thomas, R. L., \& Heck, D. G. Conditioning and retention of defensive burying as a function of elavil and thorazine injection. Bulletin of the Psychonomic Society, 1981, 17, 107-110.

Davis, S. F., Whiteside, D. A., Heck, D. G., Dickson, V. A., \& Tramill, J. L. Defensive burying: A cross-species replication and extension. Bulletin of the Psychonomic Society, 1981, 7, 45-47.

DeGhetT, V. J. Hierarchical cluster analysis. In P. W. Dolgan (Ed.), Quantitative ethology. New York: Wiley, 1978.

Hudson, B. B. One-trial learning in the domestic rat. Genetic Psychology Monographs, 1950, 41, 99-145.

Maggio, J. C., \& HARDER, D. B. Genotype and environment interactively determine the magnitude, directionality, and abolition of defensive-burying in mice. Animal Learning \& Behavior, 1983, 11, 162-172.

MCKIm, W. A., \& LETT, B. T. Spontaneous and shock-induced burying in two strains of rats. Behavioral \& Neural Biology, $1979,26,76-80$.

Misslin, R., \& Ropartz, Ph. Responses in mice to a novel object. Behaviour, 1981, 78, 169-177.

Modaresi, H. A. Defensive behavior of the rat in a shock-prod situation: Effects of the subject's location preference. Animal Learning \& Behavior, 1982, 10, 97-102.

Owings, D. H., Borchent, M., \& Virainia, R. The behavior of California ground squirrels. Animal Behaviour, 1977, 25, 211-230.

Owinas, D. H., \& Coss, R. G. Snake mobbing by California ground squirrels: Adaptive variation and ontogeny. Behaviour, 1978, 62, 50-69.

Pinel, J. P. J., Gorzalka, B. B., \& Ladak, F. Cadaverine and putrescine initiate the burial of dead conspecifics by rats. Physiology \& Behavior, 1981, 27, 819-824.

Pinel, J. P. J., \& Treit, D. Burying as a defensive response in rats. Journal of Comparative and Physiological Psychology, 1978, 92, 708-712. 
Pinel, J. P. J., \& Treit, D. Conditioned defensive burying in rats: Availability of burying materials. Animal Learning \& Behavior, 1979, 7, 392-396.

Pinel, J. P. J., Treit, D., Ladak, F., \& Maclennan, A. J. Conditioned defensive burying in rats free to escape. Animal Learning \& Behavior, 1980, 8, 447-451.

Pinel, J. P. J., Treit, D., \& Wilkie, D. M. Stimulus control of defensive burying in the rat. Learning \& Motivation, 1980, 11, 150-163.

TARTe, R. D., \& Oberdieck, F. Conditioned defensive burying in rats as a function of pre-exposure and strain. Psychological Record, 1982, 32, 101-107.

Treit, D., Terlecki, L. J., \& Pinel, J. P. J. Conditioned defensive burying in rodents: Organismic variables. Bulletin of the Psychonomic Society, 1980, 16, 451-454.

Whillans, K. V., \& Shetrleworth, S. J. Defensive burying in rats and hamsters. Animal Learning \& Behavior, 1981, 9, 357-362.

\section{APPENDIX}

The height and difference-score means for each strain were converted to the distance measure scale so that each of the three measures would have equal weight in the combined measure (the choice of which scale to convert to was arbitrary). The following formula was used for the conversion of heights to the distance scale:

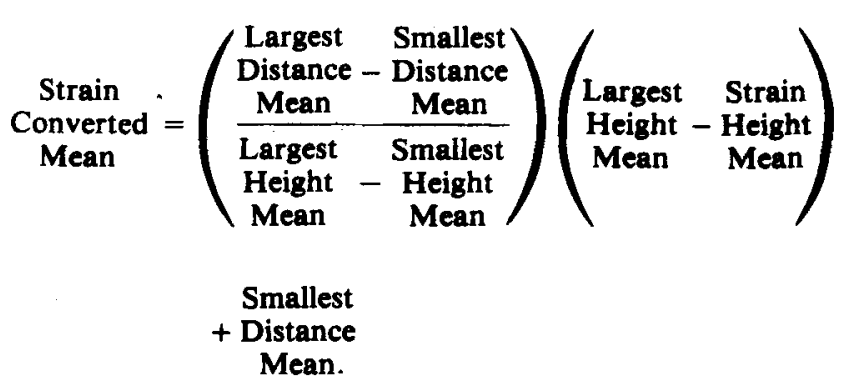

Difference scores were converted to the distance scale using the same formula (replace "height" with "difference score"). This formula compensates for the difference in the size of the ranges of scores between the measures, adjusts for the fact that better scores are larger on the height and difference-score measures but smaller on the distance measure, and gives all of the measures a common starting point. It, therefore, preserves the relationships between the strain means on the original scale.

The combined measure value for each strain was then simply the average of the distance, converted height, and converted difference-score means for that strain.

(Manuscript received April 28, 1983;

revision accepted for publication August 10, 1983.) 